\title{
Crashing of efficient stochastic bubbles
}

\author{
Aloisio Araujo ${ }^{\mathrm{a}, \mathrm{b}}$, Juan Pablo Gama ${ }^{\mathrm{a}, \mathrm{c}, *}$, Mario Rui Pascoa ${ }^{\mathrm{d}}$ \\ ${ }^{a}$ IMPA, Rio de Janeiro, Brazil \\ ${ }^{b}$ FGV EPGE, Rio de Janeiro, Brazil \\ ${ }^{c}$ Department of Economics, Federal University of Minas Gerais, Belo Horizonte, Brazil \\ ${ }^{d}$ University of Surrey, Guildford, U. K.
}

\begin{abstract}
Efficiency is not commonly related to the crash of bubbles. However in the presence of wary agents, infinite-lived agents that are worried about distant losses, efficient bubbles may occur and, in a stochastic setting, these bubbles can crash. In this paper we characterize the Arrow-Debreu (AD) price and establish the relationship between the agents' concern about distant losses and the existence of pure charges in the $\mathrm{AD}$ price. We show that this pure charge induces efficient bubbles in the positive net-supply assets that complete the markets and that, as we enter some sub-tree, that pure charge may no longer present in the $\mathrm{AD}$ price for the sub-economy, implying the crash of the bubble. Finally, we give an example in which there is an efficient bubble with infinitely many crashes.

Keywords: crashing, efficient bubbles, complete markets, stochastic economies
\end{abstract}

\section{Introduction}

Rational bubbles have been extensively studied since the late 70's pioneering work by Blanchard (1979) (see [1) and Blanchard and Watson (1982) (see [2]). Santos and Woodford (1997) (see [3]) made a theoretical and systematic study of rational bubbles in a general equilibrium model with finite and infinite-lived agents. However, efficient bubbles in positive net-supply assets seemed to be

\footnotetext{
W We gratefully acknowledge the financial support from CAPES, CNPq and FAPERJ in Brazil and from FCT/FEDER (project PTDC/IIM-ECO/5360/2012). This study was financed in part by the Coordenao de Aperfeioamento de Pessoal de Nvel Superior - Brasil (CAPES) - Finance Code 001. We also acknowledge the 16th SAET Conference and the audience of the mathematical economic seminar at IMPA.

* Corresponding author
Preprint sil of Mathematical Economics
Email address: jpgamat@impa.br (Juan Pablo Gama)

July 28, 2019
} 
ruled out by the same portfolio constraints that avoided Ponzi schemes. Araujo, Novinski and Pascoa (2011) (see 4]) showed that this is no longer the case when standard impatience assumptions do not hold. More precisely, if agents are wary, that is, not willing to neglect losses at distant dates, the efficient allocations may be implemented by trading positive net supply assets with speculative prices. The drawback of this surprising result is that it was established in a deterministic setting, where the occurrence of a bubble implies that it will be present at all dates. Araujo, Gama, Novinski and Pascoa (2019) (see [5]) showed how it can be implemented sequentially these efficient allocations in economies with money or other long-lived assets with taxes.

In a stochastic economy, bubbles can burst some time later, as we enter some sub-tree where the reason for the occurrence of the bubble does not prevail. In this paper we address whether efficient bubbles generated by wariness may burst in a stochastic economy. Wariness is a lack of impatience that consists in neglecting distant gains but not distant losses. The lack of impatience can be interpreted in terms of ambiguous beliefs, in the sense of Gilboa and Schmeidler (1989) (see [6]) and Schmeidler (1989) (see [7]), making the agents worried about their losses among the different dates and state of nature. And, as secondary objective, we provide an analysis of possible increments in asset price variation due to the crashing of bubbles in some states of nature.

We provide an example where the occurrence of a pure charge in the ArrowDebreu price may happen and then disappear, depending on whether the infimum of consumption is a cluster point of the consumption sequence or not, within the relevant sub-tree. This induces the asset price bubble or the burst of that bubble, respectively, and the bubble may even occur and then crash in infinitely many different period depending on the path. In all cases, bubbles cannot reappear in the economy.

The article is organized as follows: In section 2, we define the notation used along the article, and we define the type AD economy that we will work on. In section 3 , we characterize the superdiferential of the type of consumers that we defined. In section 4, we implement the AD allocation sequentially, and we 
analyze the existence of efficient bubbles in this framework and how they affect the variation of the asset prices, and we conclude this section with an example with a positive bubble that the probability of crashing from a very distant date onwards is positive. In section 5, we give some concluding remarks.

\section{Model}

We consider a model with a enumerable number of periods $t$, in each period, there exists a finite number of states and finite number of sucessor for each of them. In other words, the set of all the possible states can be seen as tree, and each state as a node of the tree.

Let us denote $N \subset \mathbb{N}$ a finite set that represents all the states that could occur as immediate successors of a node of the tree with $1 \in N, s^{t}=\left(s_{1}, s_{2}, \ldots, s_{t}\right) \in$ $\{1\} \times N^{t-1}$ as a node of the tree in the date $t, s^{t,-}$ as the predecessor of $s^{t}$, $s^{t,-2}=s^{t,--}$ as the predecessor of $s^{t,-}, \sigma:=\left(s_{1}, \ldots, s_{t}, \ldots\right)$ as an infinite path of the tree, $\{1\} \times N^{\infty}$ as the set of all the infinite paths $\sigma, \mathcal{N}$ as the $\sigma$-algebra induced by $\left\{\sigma: \sigma_{t}=s^{t}\right\}$ for each $s^{t} \in\{1\} \times N^{t-1}$ and for each $t \in \mathbb{N}$, and $\mathbb{P}$ as a probability measure in $\left(\{1\} \times N^{\infty}, \mathcal{N}\right)$. Therefore, $\mathbb{P}\left[\sigma_{t}=s^{t}\right]$ can be interpreted as the probability of the node $s^{t}$ to occur.

\subsection{Utility function}

The space of consumption is $\mathcal{X}:=\left\{X: \cup_{t \in \mathbb{N} \cup\{0\}}\{1\} \times N^{t} \rightarrow \mathbb{R}^{+}: \exists K>\right.$ 0 such that $\left.X\left(s^{t}\right) \leq K\right\}$ then the elements of $\mathcal{X}$ can be seen as elements of $L^{\infty}\left(\cup_{t \in \mathbb{N} \cup\{0\}}\{1\} \times N^{t}, \tilde{\mathcal{N}}, P\right)$ where $\tilde{\mathcal{N}}$ is the discrete $\sigma$-algebra in $\cup_{t \in \mathbb{N} \cup\{0\}}\{1\} \times$ $N^{t}$ and $P$ is the $\sigma$-additive measure induced by $P\left(\left\{s^{t}\right\}\right)=\mathbb{P}\left[\sigma_{t}=s^{t}\right]^{1}$

Since we are in a stochastic economy with infinite number of dates, there are several forms to implement the lack of impatience for losses. We consider a natural extension for the stochastic case of the one used in Araujo et al. (2011) (see

\footnotetext{
${ }^{1}$ The only purpose of the probability $P$ is to define properly the consumption set that we are working on.
} 
[4]), $\varepsilon$-Contamination ${ }^{2}$. It uses ambiguity aversion in the sense of Schmeidler (1989) (see [7] ${ }^{3}$ which give us a clear relationship between ambiguity in the discount factors and the importance in the worst consumptions in each path, that is,

$$
U(X):=\int_{N^{\infty} \times \mathbb{N}}^{u \circ X_{\sigma_{t}}} d \mathbb{P} \times \zeta(\sigma, t)+\int_{N^{\infty}}\left(\beta(\sigma) \inf _{t} u \circ X_{\sigma_{t}}\right) d \mathbb{P}(\sigma)
$$

where $u: \mathbb{R}_{+} \rightarrow \mathbb{R}$ is a differentiable, strictly increasing and strictly concave function, $\zeta_{t}$ is a measure in $\mathbb{N}$ that can be interpreted as the discounted factors for each state, and $\beta$ is a $\mathcal{N}$-measurable non-negative function. As can be observed in the form of the utility function, the agents worry about their consumption by considering all the possible paths in the tree.

Agents with this behavior worry about every consumption in each period if the discounted factors $\zeta$ are strictly positive, and if the probability of each state $s^{t}, \mathbb{P}\left[\sigma_{t}=s^{t}\right]$ is also positive. However, they are also concern about the worst possible outcome of each path. Differently to Araujo et al. (2019) [5], there is ambiguity not just only on the discounted factors, in this case there exists also among all the paths among of the tree.

The left part of the utility function is the standard discounted utility function in stochastic economies. The right part is the $\mathbb{P}$-average among all possible paths of the worst consumption.

A non-constant $\beta$ implies that an agent consistent with Equation 1 has different concerns about distant losses depending on the paths of the tree which is completely intuitive from the decision maker point of view. Therefore it causes that $\beta: N^{\infty} \rightarrow \mathbb{R}_{+}$is a nonnegative, bounded and not necessarily constant function of the infinite paths.

\footnotetext{
${ }^{2}$ In the deterministic case, given a probability measure $\mu$ and $\varepsilon \in[0,1)$, let $\nu_{\epsilon}$ be the convex capacity defined by $\nu(A)=(1-\varepsilon) \mu(A)$ for $A \varsubsetneqq \mathbb{N}$ and $\nu_{\varepsilon}(\mathbb{N})=1$. This is called the $\varepsilon$-contamination capacity with respect to $\mu$. We can rewrite the Choquet integral with respect of $\nu_{\varepsilon}$ as $U(x)=(1-\varepsilon) \int u \circ x d \mu+\varepsilon \inf u \circ x$.

${ }^{3} \mathrm{~A}$ preference defined in a set of acts in the Ascombe-Aumann framework is ambiguity averse if $1 / 2(f+g) \succsim f, g$ for all $f, g$ acts. In the context of Schmeidler (1989) (see [7]), it is equivalent to the capacity being convex.
} 


\subsection{Arrow-Debreu Equilibrium}

Consider an economy with $I$ consumers. Each consumer $i$ is characterized by an endowment allocation $\left\{W_{s^{t}}^{i}\right\}_{s^{t}} \in L_{+}^{\infty}\left(\cup_{t \in \mathbb{N} \cup\{0\}}\{1\} \times N^{t}, \tilde{\mathcal{N}}, P\right)$ such that $\left\{W_{s^{t}}^{i}\right\}_{s^{t}} \ggg 0^{4}$ and a preference that admits a representation consistent with Equation 11 on $L_{+}^{\infty}\left(\cup_{t \in \mathbb{N} \cup\{0\}}\{1\} \times N^{t}, \tilde{\mathcal{N}}, P\right)$.

Definition 1. An Arrow-Debreu equilibrium is a couple $\left(\pi,\left(x^{i}\right)_{i=1}^{I}\right)$ such that, for all $i, x^{i}$ maximizes $U^{i}$ on $B_{A D}\left(\pi, W^{i}\right):=\left\{x \geq 0: \pi(x) \leq \pi\left(W^{i}\right)\right\}$, $\sum_{i=1}^{I}\left(x^{i}-W^{i}\right)=0$ and $\pi \in L_{+}^{\infty *}\left(\cup_{t \in \mathbb{N} \cup\{0\}}\{1\} \times N^{t}, \tilde{\mathcal{N}}, P\right)$ with $\pi \neq 0$.

Since the weight of the infimum each path is completely negligible for all agents, this type of patience is compatible with the hypotheses imposed by Bewley (see [8]) and Mas-Colell et al. (see [9]) to ensure existence of equilibrium in economies with $L_{+}^{\infty}\left(\cup_{t \in \mathbb{N} \cup\{0\}}\{1\} \times N^{t}, \tilde{\mathcal{N}}, P\right)$ as consumption set. The price of this economy is an element of its dual, that is, $b a_{+}\left(\cup_{t \in \mathbb{N} \cup\{0\}}\{1\} \times N^{t}, \tilde{\mathcal{N}}, P\right)$ the set of the finitely additive measures in the set of nodes.

Theorem 1 (Bewley's Existence Theorems [8]).

(T1) Consider an economy with a finite number of agents where each agent has complete, convex, transitive monotonous, norm continuous and Mackey usc preferences in $l_{+}^{\infty}$, and $W^{i} \ggg 0$, then there exists an $A D$ equilibrium with prices in $b a_{++}$.

(T2) Under the additional assumption of Mackey lsc, $\pi \in l_{++}^{1}$.

Therefore, if $\left(\pi,\left(x^{i}\right)_{i=1}^{I}\right)$ is an $\mathrm{AD}$ equilibrium where $U^{i}$ is a concave function that satisfies the condition of (T1), $\left.\pi \in \partial U^{i}\left(x^{i}\right)\right|^{5}$ for all $i$.

In the next section, we will prove some important properties of the pure charges in the supergradient of the optimal solution of an agent consistent with

\footnotetext{
${ }^{4} x \geq 0$ means $x_{s^{t}} \geq 0$ for all $s^{t}, x>0$ means $x \geq 0$ and $x \neq 0, x \gg 0$ means $x_{s^{t}}>0$ for all $s^{t}$, and $x \ggg 0$ means that there exists $\varepsilon>0$ such that $x_{s^{t}} \geq \varepsilon$ for all $s^{t}$.

${ }^{5}$ For any concave function $U: B \rightarrow \mathbb{R}$ where $B$ is a Banach space, $\partial U(x):=$ $\left\{\pi \in B^{*}: U(y)-U(x) \leq \pi(y-x) \forall y \in B\right\}$ is the superdifferential of $U$ at $x$, and $\pi \in U(x)$ is a supergradient of $U$ at $x$.
} 
Equation 1. These properties will be extremely relevant to establish the relationship between the pure charges and the existence of efficient bubbles in stochastic economies.

\section{Characterization of the Super-differential of the Utility Function}

Since the utility function considered has positive weight on the infimum consumption in each path, a wary agent will be concerned about the infimum consumption in each path if the infimum is never attained. Therefore, there is a positive pure charge in the superdifferential in the optimal allocation. The following result is an extension of the deterministic case analyzed in Araujo et al. (2011) 4].

Proposition 1. Let $X \ggg 0, \underline{X}: N^{\infty} \rightarrow \mathbb{R}_{++}$such that $\underline{X}_{\sigma}=\inf _{t} X_{\sigma_{t}}$. For $U$ given by Equation 1 with $u \in C^{1}(0, \infty)$ and $\pi \in L^{\infty *}\left(\cup_{t \in \mathbb{N} \cup\{0\}}\{1\} \times N^{t}, \tilde{\mathcal{N}}, P\right)$. We have that $\pi$ can be written as

$$
\begin{aligned}
\pi Y= & \sum_{s^{t}} \int_{\left[\sigma: \sigma_{t}=s^{t}\right]} u^{\prime} \circ X_{\sigma_{t}} \zeta_{t} d \mathbb{P}(\sigma) Y_{s^{t}}+\int_{A_{1}} \beta(\sigma) u^{\prime} \circ \underline{X}_{\sigma} \operatorname{LIM}_{\sigma}\left(Y_{\sigma}\right) d \mathbb{P}(\sigma)+ \\
& \sum_{t_{1} \leq \cdots \leq t_{K}} \int_{A_{2, t_{1}, \ldots, t_{K}}^{K}} \beta(\sigma) u^{\prime} \circ \underline{X}_{\sigma}\left(\sum_{k=1}^{K} \alpha_{2}(\sigma, k) Y_{\sigma^{t_{k}}}\right) d \mathbb{P}(\sigma)+ \\
& \sum_{t_{1} \leq t_{2} \leq . .} \int_{A_{3, t_{1}, t_{2}}, \ldots} \beta(\sigma)\left(u^{\prime} \circ \underline{X}_{\sigma}\right)\left(\sum_{k=1}^{\infty} \alpha_{3}(\sigma, k) Y_{\sigma^{t_{k}}}+\right. \\
& \left.\alpha_{3}(\sigma, \infty) \operatorname{LIM}_{\sigma}\left(Y_{\sigma}\right) \beta(\sigma)\right) d \mathbb{P}(\sigma),
\end{aligned}
$$

where $Y \in L^{\infty}\left(\cup_{t \in \mathbb{N} \cup\{0\}}\{1\} \times N^{t}, \tilde{\mathcal{N}}, P\right)$ and

- $A_{1}:=\left[\sigma: \underline{X}_{\sigma}<u\left(X_{\sigma^{t}}\right) \forall t \in \mathbb{N}\right]$,

- $A_{2, t_{1}, \ldots, t_{k}}^{K}:=\left[\sigma: \inf _{t} u \circ X_{\sigma_{t}}=u\left(X_{\sigma^{t_{k}}}\right) \forall k=1, \ldots, K \wedge u\left(X_{\sigma_{t}}\right) \geq\right.$ $u\left(X_{\sigma^{t_{k}}}\right)+\varepsilon \forall t \neq t_{1}, \ldots, t_{K}$ for some $\left.\varepsilon>0\right], A_{2}:=\bigcup_{K}\left(\bigcup_{t_{1} \leq \cdots \leq t_{K}} A_{2, t_{1}, \ldots, t_{k}}^{K}\right)$,

- $A_{3, t_{1}, t_{2} \ldots}:=\left[\sigma: \inf _{t} u \circ X_{\sigma_{t}}=u\left(X_{\sigma^{t_{k}}}\right) \forall k \geq 1 \wedge u\left(X_{\sigma_{t}}\right)>u\left(X_{\sigma^{t_{k}}}\right) \forall t \neq\right.$ $\left.t_{1}, t_{2}, \ldots\right], A_{3}:=\bigcup_{t_{1} \leq t_{2} \leq \ldots} A_{3, t_{1}, t_{2} \ldots}$

- $\alpha_{2}(\sigma, k) \geq 0 \forall k=1, \ldots, K$ and $\sum_{k=1}^{K} \alpha_{2}(\sigma, k)=1, \forall \sigma \in\{1\} \times N^{\infty}$,

- $\alpha_{2}(\sigma, k), \alpha_{2}(\sigma, \infty) \geq 0 \forall k \in \mathbb{N}$ and $\sum_{k=1}^{\infty} \alpha_{3}(\sigma, k)+\alpha_{3}(\sigma, \infty)=1, \forall \sigma \in$ $\{1\} \times N^{\infty}$ and 
- $\left\{\mathrm{LIM}_{\sigma}\right\}_{\sigma \in A_{1} \cup A_{3}}$ is a collection of generalized limit $\oint^{6}$ such that it is a measurable function in $\left(\{1\} \times N^{\infty}, \mathcal{N}\right)$,

if and only if $\pi \in \partial U(X)$.

The proof can be found in Appendix A.

Note that, in our framework, it is not enough to analyze the cases in which the infimum is never attained, or to analyze the cases in which it is always attained in finite or infinite number of times only as in Araujo et al. (2011) 44. Moreover, any allocation, the set of paths can be separated in three main sets: the ones in which the infimum is never attained (denoted $A_{1}$ ), the ones in which the infinimum is attained only in a finite number of periods (denoted $A_{2}$ ), and the ones in which the infimum is attained in an infinite number of periods (denoted $A_{3}$ ). In each set, the super-differential has different properties. Under $\left.\beta(\sigma)\right|_{A_{1}} \gg 0 \mathbb{P}$-a.c.7 $]^{7}$ in $A_{1}$ and $\mathbb{P}\left[A_{1}\right]>0$, there is a positive pure charge in the supergradient that is concerned about paths in $A_{1}$ only. The pure charge of the supergradient component in $A_{2}$ does not exists since the infimum of $X$ is attained a finite number of times only. For the latter, there are supergradients without pure charges component that is concerned about $A_{3}$ only (because $\alpha(\sigma, \infty)=0 \mathbb{P}$ a.c. in $A_{3}$ for that supergradient), but, under $\left.\beta(\sigma)\right|_{A_{3}} \gg 0 \mathbb{P}_{-}$ a.c. in $A_{3}$ and $\mathbb{P}\left[A_{3}\right]>0$, there are supergradients with a positive pure charge component that has that property (because $\alpha(\sigma, \infty)>0$ for a positive measure of paths in $A_{3}$ for that supergradient).

From Proposition 1, we know that the existence of pure charges in the in the super-gradient is strongly related to the infimum being attained in a finite number of periods in each path. However, in the case in which the infimum is attained an infinite number of times, there are supergradients with no pure charge component, but there are also supergradients with a pure pure charge taking nonnegative values in the path in which the infinimum is attained.

\footnotetext{
${ }^{6} \mathrm{~A}$ generalized limit is a continuous, linear functional in $l^{\infty, *}, \operatorname{LIM}$, such that $\operatorname{LIM}(x)=$ $\lim _{t} x_{t}$ when it exists, and $\operatorname{LIM}(x) \in\left[\liminf x_{t} x_{t}, \lim \sup _{t} x_{t}\right]$ when it does not.

$\left.{ }^{7} x\right|_{A}$ means that $x$ is restricted to the paths contained in $A$.
} 
Remark 1. If $X \gg 0$ instead of $X \ggg 0$, there might be pure charges generated by the fact that $\left\{u^{\prime}\left(X_{s^{t}}\right)\right\}_{s^{t}}$ is not bounded. Moreover, if $\left\{\zeta_{t} \mathbb{P}\left[\sigma_{t}=s^{t}\right] u^{\prime}\left(X_{s^{t}}\right)\right\}_{s^{t}}$ is not in $l^{1}, \partial U(X) \nsubseteq l^{1}$.

\section{Bubbles in sequential implementation of an $\mathrm{AD}$ equilibrium}

Let us now define a sequential economy with one good in each state, which is considered as a numéraire, and $|N|$ Long-Lived assets with nonnegative returns $\left\{R_{j, s^{t}}\right\}_{s^{t}} \in L_{+}^{\infty}\left(\cup_{t \in \mathbb{N} \cup\{0\}}\{1\} \times N^{t}, \tilde{\mathcal{N}}, P\right)$ for each $j$. Additionally, each asset has a short-sales constraints. Each agent has an initial nonnegative amount of each asset $z_{j, 0}^{i} \geq 0$, and an initial endowment $\left\{\omega_{s^{t}}^{i}\right\}_{s^{t}, i} \geq 0$ where $\omega_{s^{t}}^{i}=$ $W_{s^{t}}^{i}-\sum_{j} R_{j, s^{t}} z_{j, 0}^{i}$ for each $s^{t}$. Therefore, the consumer's problem is to find an optimal portfolio $\left\{z_{s^{t}}\right\}_{s^{t}} \in \mathbb{R}^{\infty}$ to maximize $U^{i}$ subject to the sequential budget constraints

$$
\begin{aligned}
x_{s^{t}}-\omega_{s^{t}} & \leq q_{s^{t}}\left(z_{s^{t,-}}-z_{s^{t}}\right)+R_{s^{t}} z_{s^{t,-}}, \\
z_{s^{t}} & \geq-K_{s^{t}},
\end{aligned} \text { for all } s^{t}, t \geq 1,
$$

where $x_{s^{t}}$ is the consumption of the numéraire, $\omega_{s^{t}}$ is the endowment of the numéraire, $q_{1, s^{t}}, \ldots, q_{J, s^{t}}$ are the prices of the assets, $z_{s^{t}}$ is the assets' allocation on the node $s^{t}$, and $\left\{K_{s^{t}}\right\}_{s^{t}}$ is nonnegative and bounded from above.

Our goal is to analyze efficient bubble in this economy. By efficient bubbles we mean the bubbles that occur in the implementation of an $\mathrm{AD}$ equilibrium $\left(\pi, x^{i}\right)$ of Subsection 2.2 in stochastic sequential economies as the ones described in the current section with a bounded short-sale constraint adjusted to include the optimal allocation in the budget constraint of the agent. When agents are not impatient, long-run improvement opportunities are not ruled out by bounded short-sales constraints, see Araujo et al. (2019) [5], that is, the existence of a portfolio that allows the agent to increase his/her utility by increasing the long run consumption over-saving the assets to do st 8

\footnotetext{
${ }^{8}$ Take any non-negative real sequence $z$ and let $x \equiv x(z, i)$. Suppose consumer $i$ replaces $z_{t}^{i}$ by $z_{s^{t}}^{i}+h z_{s^{t}}$, with $h>0$, from some date $n$ onward. As we move on the right along this
} 
To avoid the long-run improvement opportunities mentioned above, we must impose an additional constraint such as taxes as in Araujo et al. (2019) [5] argued. Another form to avoid them is to impose some type of P-constrain $t^{9}$ mentioned in Araujo et al. 4 as a transversality condition in the budget constraint to it prohibit the existence of such opportunities. The necessity of this type of constraints is a direct consequence of the lack of impatience since they would have incentives to save large quantities of wealth in form of assets to avoid the worst states in distant dates.

Since in this article we have more interest in analyze efficient bubbles than to find different ways of implementing efficient allocations, we will assume that each agent has some type of $P$-constraint that allows us to implement efficient allocation. However, note that the implementation with money and taxes is also possible even in stochastic economies with agents concerned about the average of the worst possible outcomes. To implement this efficient allocations sequentially with taxes or with a $P$-constraint, it is required that the market structure is complete (in the sense of $\operatorname{span}\left(R_{s^{t}}+q_{s^{t}}\right)=N$ ) in each node. In the case of taxes in a complete market economy, the taxes would not be defined by the limsup as in the deterministic case, it would be defined by the average of the limsup in the paths since the taxes depend indirectly on how the agents concern about the distant losses.

Since there is a strong relationship between the AD prices and the supergradients of the equilibrium allocations, we can characterize the $\mathrm{AD}$ price based on the equilibrium allocation. The next section explores the relationship between the existence of efficient bubbles and the AD prices.

direction, we hoard more at date $n$ and at subsequent dates for which $\omega_{s^{t}}^{i}>x_{s^{t}}$, in order to consume more at subsequent dates where $\omega_{s^{t}}^{i}<x_{s^{t}}$. There would be a utility gain by doing so if the right derivative were positive. Now, the right derivative is the minimum of the values that all supergradients may take at this direction and, therefore, such improvement strategy is ruled out if there is a supergradient $T=\mu+\nu$ for which $T(c(z(n))) \leq 0$.

${ }^{9} \lim _{t} \sum_{s^{t}} \mu_{s^{t}} q_{s^{t}} z_{s^{t}} \geq \nu\left(x(z, i)-\omega^{i}\right)$ where $\nu$ is the capacity of the AD price. 


\subsection{Characterization of Efficient Bubbles and their Crashing}

Based on Santos and Woodford [3], a rational bubble at a node $s^{t}$ is defined as difference between the price of the asset in this state and the fundamental value of it

$$
q_{s^{t}}-\frac{1}{a_{s^{t}}} \sum_{r=t+1}^{\infty} \sum_{s^{r,-(r-t)}=s^{t}} a_{s^{r}} R_{s^{r}} \geq 0,
$$

where $\left\{a_{s^{t}}\right\}_{s^{t}}$ are the state prices that exists due to the lack of arbitrage in the economy.

Due to the Euler equations applied in an optimal efficient allocation $\left(x^{i}\right)_{i} \gg$ 0 , the $L_{++}^{1}\left(\cup_{t \in \mathbb{N} \cup\{0\}}\{1\} \times N^{t}, \tilde{\mathcal{N}}, P\right) \equiv \ell_{++}^{1}$ component of the $A D$ price, $\left\{\mu_{s^{t}}\right\}_{s^{t}} 10$ satisfies that there is a constant $\alpha>0$ such that $\mu_{s^{t}}=\alpha a_{s^{t}}$ for any $s^{t}$. Therefore, without loss of generality it can be assumed that the deflator are $\left\{\mu_{s^{t}}\right\}_{s^{t}}$. Moreover, we have that

$$
\mu_{s^{t}} q_{s^{t}}-\sum_{r=t+1}^{\infty} \sum_{s^{r,-(r-t)}=s^{t}} \mu_{s^{r}} R_{s^{r}}=\lim _{r \rightarrow \infty} \sum_{s^{r,-(r-t)}=s^{t}} \mu_{s^{r}} q_{s^{r}} .
$$

Therefore, the existence of efficient bubbles in a node $s^{t}$ depends on the asymptotic behavior of the subtree that is generated by this node which implies that if a bubble crashes at some node $s^{t}$, a bubble can not reappear in the economy in any sucessor of $s^{t}$.

Proposition 2. For any efficient allocation implemented in a sequential economy with a complete set of Long-Lived assets. If there exists one node $s^{t}$ and one asset $j$ in which there is no bubble for the asset price $q_{s^{t}}^{j}$ then there is no bubble for the asset price $q_{s^{r}}^{j}$ for any state $s^{r}$ sucessor of $s^{t}$.

Then, after the crashing of a bubble of any asset at the node $s^{t}$, there is no possible reappearance of the bubble in the subtree generated by $s^{t}$. However, it does not mean that there is no bubble in the economy in other subtrees that are not generated by $s^{t}$.

\footnotetext{
${ }^{10}$ Therefore, the MRS for each pair of nodes $s^{t}$ and $s^{t^{\prime}}$ in the efficient allocation is equivalent to $\mu_{s^{t}} / \mu_{s^{t^{\prime}}}$.
} 
Now, let us analyze conditions in which it is possible the existence of bubbles. To do so, we will establish a relationship between bubbles and the existence of pure charges in the super-gradient of the utility function.

Since the pure charges are related to the agents' concerns of the worst events and the bubble is strongly related to the asymptotic behavior of the asset price, the existence of bubbles in this environment seems to be strongly related with the asymptotic behavior of the agents and the existence of the pure charges in the optimal super-gradient. Moreover, we have that implementing the efficient allocations in a sequential economy we have that

$$
\tilde{\nu}^{i}\left(X^{i}-\omega^{i}\right)-\nu\left(X^{i}-\omega^{i}\right)=\sum_{j}\left(z_{j, 0}^{i}\left(\mu_{1} q_{j, 1}-\sum_{s^{t}} \mu_{s^{t}} R_{j, s^{t}}-\nu\left(R_{j}\right)\right)\right)
$$

where $\tilde{\nu}$ is the capacity that takes the highest value on the direction of the net trade, and $\nu$ is the capacity of the AD price. Therefore $\mu_{1} q_{j, 1}=\sum_{s^{t}} \mu_{s^{t}} R_{j, s^{t}}+$ $\nu\left(R_{j}\right)$ if $x_{t}^{i}-\omega_{t}^{i}$ converges for any $i$, and $\mu_{1} q_{j, 1}>\sum_{s^{t}} \mu_{s^{t}} R_{j, s^{t}}+\nu\left(R_{j}\right)$ for at least one asset $j$ and $\mu_{1} q_{j, 1} \geq \sum_{s^{t}} \mu_{s^{t}} R_{j, s^{t}}+\nu\left(R_{j}\right)$ for every $j$ if $x_{s^{t}}^{i}-\omega_{s^{t}}^{i}$ doesn't converge for some $i$ and $R \geq 0$.

Proposition 3. Given an efficient allocation such that $\left(x^{i}\right)_{i} \gg 0$. If $\partial U^{i}\left(x^{i}\right) \subseteq$ $\ell^{1}$ for all agent $i$, there is no bubble for any asset in positive net supply.

Therefore, to find a bubble in assets in positive net supply, we must have that $\partial U^{i}(x) \nsubseteq \ell^{1}$. This result is a consequence of Santos and Woodford (1997) (see [3]). Moreover, Equation 3 ensures that the converse is also true under some circumstances.

Proposition 4. Given an efficient allocation such that $\left(x^{i}\right)_{i} \gg 0$. If

1. $x_{s^{t}}^{i}-\omega_{s^{t}}^{i}$ does not converge in a set of positive measure in $\mathbb{P}$ for some $i$, or

2. $\nu(R)>0$, then there is a bubble for some asset $j$ in positive net supply. 
However for nodes different than 1 the analysis can not be done for each asset separately. We will analyze the existence of bubbles for the hole set of assets, this means that even in presence of a bubble in a node $s^{t}$ with $t \neq 1$ we could have no bubble for some assets even in the presence of bubbles for every asset $j$ at some node $s^{r}$ predecessor of $s^{t}$.

The following propositions study the condition for the existence or nonexistence of a bubble in the assets at a node $s^{t}$. This conditions are strongly related to the sets $A_{1}, A_{2}$ and $A_{3}$ defined in Proposition 1

Proposition 5. For any efficient allocation with $\left(x^{i}\right)_{i} \gg 0$ and any state $s^{t}$, if there is one agent $i$ such that

1. $\left.x^{i}\right|_{\left[\sigma: \sigma_{t}=s^{t}\right]} \ggg 0$, and

2. all paths that contain $s^{t}$ attain the infimum consumption in a finite number of dates $\mathbb{P}$ almost certainly in the set $\left\{\sigma: \sigma_{t}=s^{t}\right.$ and $\left.\beta(\sigma)>0\right\}$, then there is no bubble for any asset at the node $s^{t}$.

Note that this proposition is strongly related with the set $A_{1}$ in Proposition 1 for $U=U^{i}$ and the comments that follow this result.

This implies that, to have a crashing of a bubble at a node $s^{t}$, it is enough to ensure that the infimum is attained in a finite number of dates for all the paths that contain $s^{t}$, and that there is a set of nodes with a positive probability in which the pure charge is positive.

Let us expose some conditions in which we can ensure a positive bubble at a node $s^{t}$. These conditions are related to the infimum not being attained in finite time.

Proposition 6. For any efficient allocation with $\left(x^{i}\right)_{i} \gg 0$ and any state $s^{t}$, if there exists one agent $i$ and a subset of paths $A^{i}$ with the following properties

1. $\left.x^{i}\right|_{\left[\sigma: \sigma_{t}=s^{t}\right]} \ggg 0$,

2. $A^{i}$ has positive $\mathbb{P}$-probability,

3. any $\sigma$ in $A^{i}$ satisfies that $\sigma_{t}=s^{t}$, and 
4. for each $\sigma$ in $A^{i}$, the infimum of $\left\{X_{\sigma^{r}}^{i}\right\}_{r \in \mathbb{N}}$, the sequence of consumption of the agent $i$ in the path $\sigma$, is a cluster point never attained.

Then, there is a bubble at the node $s^{t}$ if (1) there exists at least one asset in positive net supply such that $\nu\left(R^{j, s^{t}+}\right)>0$ or (2) the net trade $x^{i}-\omega^{i}$ are not convergent in $A^{i}$.

From Proposition 5 and 6 and their proof, the existence of efficient bubbles in this economy is related to the existence of positive pure charges in the supergradient of the agents as it was mentioned in Araujo et al (2011) (see 4]) in deterministic economies. In this case, Proposition 1 allows us to know the conditions that must be satisfied to ensure the existence of pure charges in the superdifferential of each agent.

Note that, in Proposition 6, the set $A^{i}$ is a subset of $A_{1}$ defined in Proposition 1 for $U=U^{i}$.

Similar to the deterministic case, the desire of the WARY agent of increasing their consumption in the worst events in the subtree that contains the node $s^{t}$, produces a pure charge in the AD price that imply the existence of bubbles for the set of assets at the node.

\subsection{Variation of prices in presence of Efficient Bubbles and Crashing}

If, at some period of time, there is a bubble in some nodes, and there is not in some other nodes, it means that, before that, the bubble crashes in at least one node. Therefore, if we analyze the asset prices in these subtrees, we can analyze the changes in the asset the variation of the assets prices in short and long run.

Under the conditions described in Proposition 5 and 6 , it is possible to know when and where, in the tree, there is a bubble and also when it will crash. Since these bubbles increase the price above the fundamental value of the assets, the crashing of them will, naturally, increase the dispersion of the asset prices when the price's variations are analyzed for all successors $s^{t}$ of a node $s^{r}$ with a bubble in at least one asset. 
After the bubble crashes at the node $s^{t}$, the fundamental value of the asset and the market price are equal, this means that $\max _{\left\{s^{r}: s^{r,-(r-t)}=s^{t}\right\}} \mu_{s^{r}} q_{j, s^{r}}=\max _{\left\{s^{r}: s^{r,-(r-t)}=s^{t}\right.}\left\{\sum_{k>r} \sum_{s^{k,-(k-r)}=s^{r}} \mu_{s^{k}} R_{s^{k}}^{j}\right\} \rightarrow 0$ when $r \rightarrow \infty$. However if there is a path $\sigma$ such that there is always a bubble for the asset $j$ we have that

$$
\mu_{\sigma_{t}} q_{j, \sigma_{t}}=\sum_{r>t} \sum_{s^{r,-(r-t)}=\sigma_{t}} \mu_{s^{r}} R_{s^{r}}^{j}+\lim _{r} \sum_{s^{r,-(r-t)}=\sigma_{t}} \sum_{j} \mu_{s^{r}} q_{j, s^{r}}
$$

and, as we have already mentioned, there exists a relationship between the existence of bubbles and the pure charges in the super-gradient of the agents. Therefore, by proposition 1, we have:

Proposition 7. Under the conditions of Proposition 1 and for any path $\sigma$, the component in Equation 4 that defines the bubble of any asset $j$ at the node $\sigma_{t}$ tends to zero when $t \rightarrow \infty$.

This means that the bubble is being distributed among all nodes where the pure charge is positive, reducing its weight in the price of the asset in which the bubble is maintained.

Nevertheless it does not mean that the price would be bounded, in fact we have:

Proposition 8. Under the conditions of Proposition 6 satisfying (1') $\left.R^{j}\right|_{A_{i}} \ggg$ 0 instead of (1). For any path $\sigma \in A^{i}$, we have that

$$
\limsup _{t \rightarrow \infty}\left\{\frac{\lim _{r \rightarrow \infty} \sum_{s^{r,-(r-t)}=\sigma_{t}} \sum_{j} \mu_{s^{r}} q_{j, s^{r}}}{\mathbb{P}\left(\left[\tilde{\sigma}: \tilde{\sigma}_{t}=\sigma_{t}\right]\right)}\right\}=\beta<\infty,
$$

and there exists a path $\sigma \in A^{i}$ such that

$$
\liminf _{t \rightarrow \infty}\left\{\frac{\lim _{r \rightarrow \infty} \sum_{s^{r,-(r-t)}=\sigma_{t}} \sum_{j} \mu_{s^{r}} q_{j, s^{r}}}{\mathbb{P}\left(\left[\tilde{\sigma}: \tilde{\sigma}_{t}=\sigma_{t}\right]\right)}\right\}=\alpha>0 .
$$

And since there is a strong relationship between $\mathbb{P}\left(\left[\tilde{\sigma}: \tilde{\sigma}_{t}=\sigma_{t}\right]\right)$ and $\mu_{s^{t}}$ due to the EE, we will have: 
Corollary 1. Under the conditions exposed in Proposition 8, the bubble will tend to infinity when $t \rightarrow \infty$.

This result is also a consequence of Santos and Woodford (1997) (see [3]), and it implies in our framework that, when $t$ is large, there are large variations of prices between states due to the existence of bubbles in some nodes $s^{t}$, which make the asset prices goes to infinity in several paths, but not in everyone. Moreover, the set of paths in which the bubble crashes could have positive value.

Therefore, when there is a crashing of a bubble in the node $s^{t}$, the variations of the asset prices of a subtree that contains the node $s^{t}$ and the predecessor of $s^{t}$ tends to infinity in the long-run. Therefore, the existence of bubble is associated with high variations of the assets' price if there is a chance of the bubble to crash.

\subsection{Example}

As can be noticed through this article, there is a variety of possibilities for bubbles that can occur in the economy. The following example shows the large variety of possibilites of bubbles that can be made in this framework. It shows that bubbles can occur with positive probability even in presence of a infinite number of paths in which the bubbles crash.

Example 1. Consider an economy with two agents and two states, $N=\{1,2\}$, with utility index given by $u^{i}(x):=\ln (x) \xi_{t}^{i 1}:=1 / 2^{t}, \beta^{i}=\beta>0$ for each $i=1,2, W: \cup_{t \in \mathbb{N}}\{1\} \times N^{t} \rightarrow \mathbb{R}_{+}$given by

\footnotetext{
${ }^{11}$ In this case, each $U^{i}$ is defined in all $x \gg 0$ uniformly bounded, but the preference induced by this utility function is well-defined for $x \geq 0$ uniformly bounded. Therefore, the utility function can be extended to the case in which $U^{i}(x)=-\infty$ for all $x=0$ in at least one node.
} 
$W_{s^{t}}= \begin{cases}8+1 / 2^{t-4} & \text { if } s^{t,-(t-2)}=(1,2) \text { and } \exists k \in \mathbb{N} \text { such that } 2 k+1 \leq t \text { and } \\ & s_{2 k+1}^{t,-(t-2 k+1)}=2, \\ 9 & \text { if } s^{t}=(1,2),(1,2,1,1) \text { or } s^{t,-}=(1,2,2), \\ 10 & \text { if } t=1 \text { or } s^{t,-}=(1,2), \\ 11 & \text { otherwise if } t \text { is even, }\end{cases}$

$W_{s^{t}}^{1}=W_{s^{t}}+A_{t}$ and $W_{s^{t}}^{2}=W_{s^{t}}-A_{t}$ where $A_{t}$ is 1 if $t$ is even and $-1 / 2$ if $t$ is odd. The probability of each node $s^{t}$ to occur is symmetric with all the nodes at the date $t$, that is, that $\mathbb{P}\left[\sigma: \sigma_{t}=s^{t}\right]=1 / 2^{t-1}$ for all $s^{t} \in\{1\} \times N^{t-1}$. Let us find an $\mathrm{AD}$ equilibrium that exists in this cases because $W^{i} \ggg 0$. Given $a:\{1,2\} \rightarrow \mathbb{R}_{++}$, the consumption plan $x^{i}=a(i)\left(W^{1}+W^{2}\right)=2 a(i) W$ is optimal under the budget constraint $\pi x \leq \pi W^{i}$ when the prices are given by

$$
\begin{aligned}
& \pi x=\int \frac{x_{\sigma_{t}}}{2^{t-1} W_{\sigma_{t}}} d \mathbb{P}(\sigma)+\beta\left(x_{(1)} / 20+x_{(1,2)} / 72+\sum_{s \geq 2} \frac{x_{\tilde{\sigma}_{2 s+1}}}{2^{2 s+1}\left(8+1 / 2^{2 t-3}\right)}+\right. \\
& \left.\int_{\bigcup_{t \in \mathbb{N}}}\left[\sigma: \sigma_{2 t+1}=(1,2,1,1, \ldots, 1,2)\right] \operatorname{LIM}_{\sigma}\left(x_{\sigma}\right) d \mathbb{P}(\sigma)\right)
\end{aligned}
$$

where $\tilde{\sigma}_{2 t+1}=(1,2,1,1, \ldots, 1)$ and $a(i)=\pi\left(W^{i}\right) / \pi(W)$. One possible generalized limit is the Banach limit, $B$, which satisfies that $B\left(\left(x_{t}\right)_{t \in \mathbb{N}}\right)=\lim _{n \rightarrow \infty} 1 / n \sum_{t=1}^{n} x_{t}$ when the limit of the sequence $\left\{1 / n \sum_{t=1}^{n} x_{t}\right\}_{n}$ exists. Under this condition $\left(\pi, x^{1}, x^{2}\right)$ is an $\mathrm{AD}$ equilibrium.

For the sequential implementation with two long-lived assets, one that pays 1 in each state 1 and 0 in each state 2 , and the other one that pays 0 in each state 1 and 1 in each state 2 , we can fix the prices $q_{1} \in \mathbb{R}_{++}^{2}$ and $z_{0}^{i}$ such that $\left(x^{i}, z^{i}\right)$ is implemented sequentially which implies that Equation 3 must be satisfied, that is, $\tilde{\nu}^{i}\left(x^{i}-\omega^{i}\right)-\nu\left(x^{i}-\omega^{i}\right)=\sum_{j=1}^{2}\left(z_{j, 0}^{i}\left(\mu_{1} q_{j, 1}-\sum_{s^{t}} \mu_{s^{t}} R_{j, s^{t}}-\nu\left(R_{j}\right)\right)\right)$ where $\nu$, the capacity of the $\mathrm{AD}$ price, and $\nu^{i}$, the pure charge in the subgradient of $U^{i}$ in $x^{i}$ that takes the largest value in the net trade $x^{i}-\omega^{i}$, have the form of Equation 7. $\left\{\mu_{s^{t}}\right\}_{s^{t}}$ the $l^{1}$ component of the AD price and the no-arbitrage state prices that are defined by 


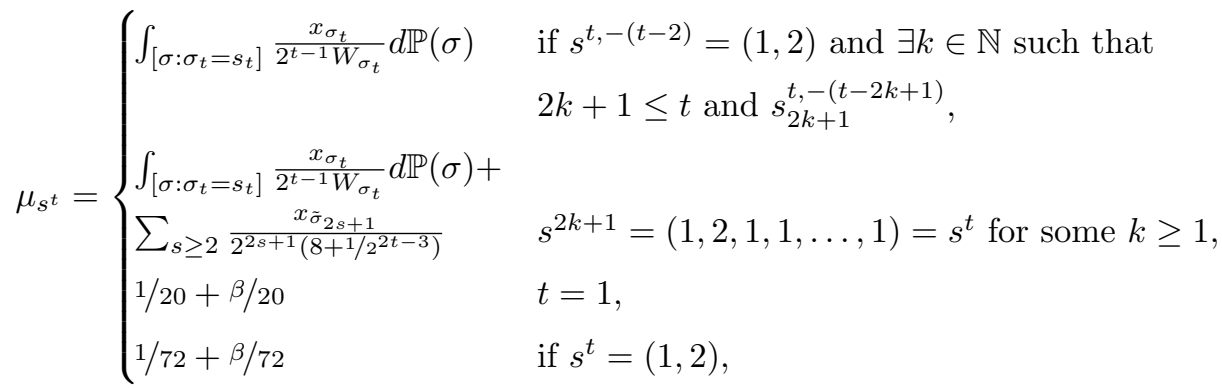

$\left\{q_{s^{t}}\right\}_{s^{t}, t \geq 2}$ is defined based on $q_{1}$ and the Euler equation $\mu_{s^{t}} q_{s^{t}}=\mu_{s^{t, 1}}\left(q_{s^{t, 1}}+\right.$ $\left.R_{s^{t, 1}}\right)+\mu_{s^{t, 2}}\left(q_{s^{t, 2}}+R_{s^{t, 2}}\right)$ for all $s^{t}$ with $t \geq 1$. To ensure optimality of the efficient allocation, it must be imposed a P-constraint, $\lim _{t} \sum_{s^{t}} \mu_{s^{t}} q_{s^{t}} z_{s^{t}} \geq$ $\nu\left(x(z, i)-\omega^{i}\right)$ where $\nu$ is the capacity of the AD price, (see Araujo et al. (2019) 4] to avoid the long-lived improvement opportunities.

As a consequence, the sequential prices $\left(q_{s^{t}}^{1}, q_{s^{t}}^{2}\right)_{s^{t}}$ satisfy the conditions exposed before, which implies the relationship between the optimal paths and the existence of bubbles in the economy.

Therefore, using the relationship between $W$ and the existence of bubble, we have that, in $s^{2}=(1,1), s^{4}=(1,2,1,2), s^{6}=(1,2,1,1,1,2), \ldots, s^{2 t}=$ $(1,2,1, \ldots, 1,2), \ldots$, the bubble crashes. And, in spite of the infinite number of paths for which the bubbles crash, the proportion of paths with positive bubble at any state is positive, in fact,

$$
\begin{aligned}
\mathbb{P}\left[\sigma: \inf _{s} X_{\sigma^{s}}<X_{\sigma_{t}} \forall t \in \mathbb{N}\right] & =\mathbb{P}\left[\sigma: \inf _{s} W_{\sigma^{s}}<W_{\sigma_{t}} \forall t \in \mathbb{N}\right] \\
& =\mathbb{P}[\sigma: \text { the bubble in the path } \sigma \text { does not crash }] \\
& =1 / 3 .
\end{aligned}
$$

This example helps us to notice that in stochastic economies, the existence of bubbles in considerably large set of paths is consistent with the crashing of bubbles in a large amount of period, for different set of paths at each time. We can say also that the existence of rational efficient bubbles, as in the deterministic case, is related to the lack of impatience of the agents. 

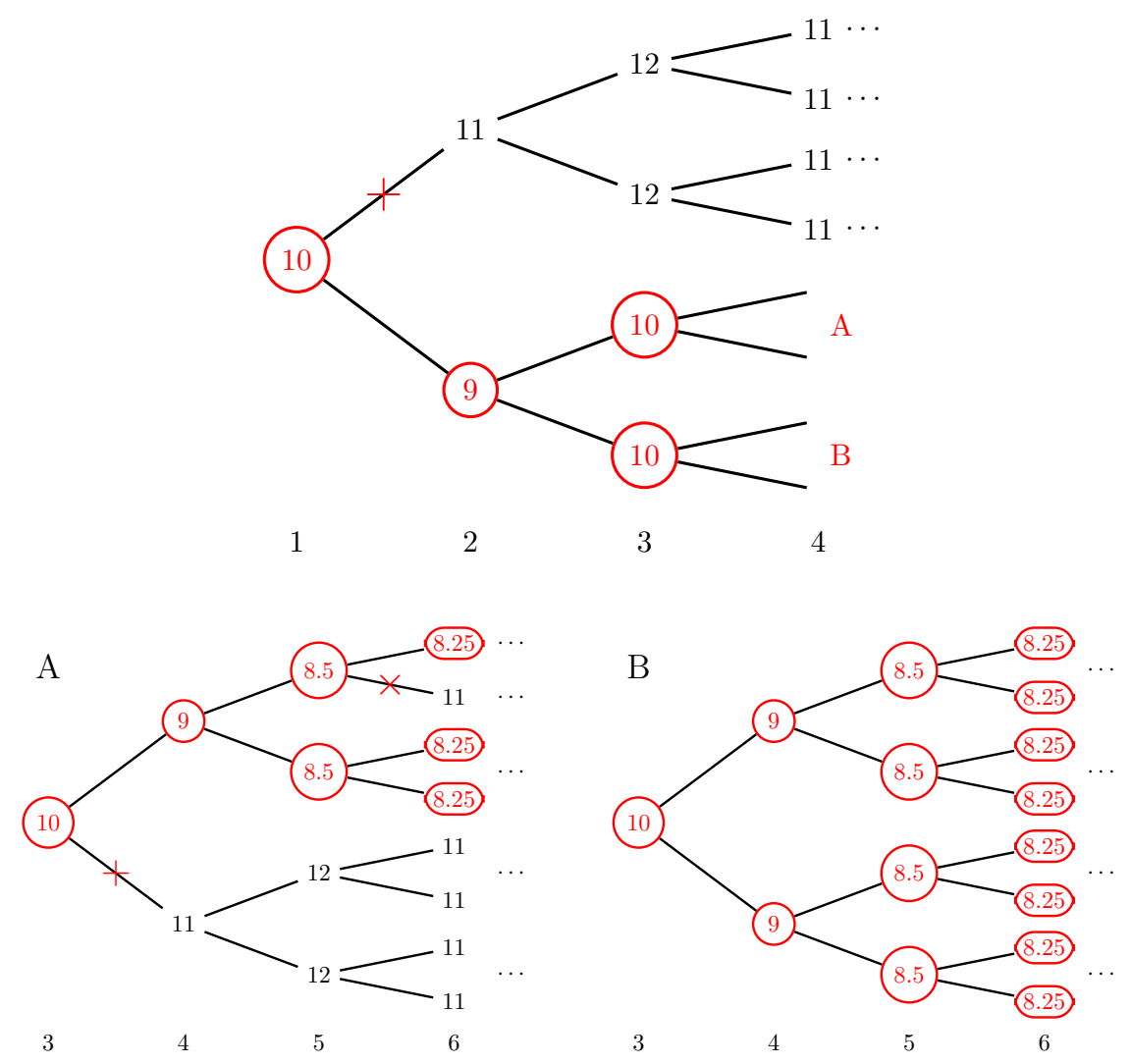

Figure 1: Distribution of $W$, existence of bubbles (with red circles) and crashing (with red $\times$ 's)

\section{Concluding Remarks}

Given that the crash of bubbles is impossible in deterministic intertemporal economies, it is natural to consider economies that allow for a large variety of bubble patterns. One of these possible settings is to consider stochastic intertemporal economies with lack of impatience. We observed that the form that agents are worried about distance losses affects the pure charges in the price of the $\mathrm{AD}$ economy and the existence of bubbles in the sequential economy. These results (Section 4.1) are independent on the specific form of considering distant losses.

When the agents are worried about distant losses in each path, it is possible 
to establish a relationship between the existence of positive pure charges in the $\mathrm{AD}$ price and the existence of bubbles, as in the deterministic case.

To do so, we considered a utility function that is compatible with the model defined by Schmeidler (see [7]) and Gilboa adn Schmeidler (1989) (see 6]) that is a generalization of the $\varepsilon$-contamination utility function.

The way we modelled impatience in a stochastic framework is also compatible with the existence of $\mathrm{AD}$ equilibrium, as in the result by Bewley (see [8]). We also noticed that for this type of utility function, the existence of a positive charge in the AD price is strongly related to the asymptotic consumption of the agents, and then, the existence of efficient bubbles in the intertemporal economy at the first date. Moreover, we proved that the relationship between bubbles and the existence of pure charges is also maintained for every node of the tree. Therefore, to analyze the existence of efficient bubbles at any node, it is enough to know the efficient consumption patterns of the agents. We also analyzed the variation of the assets' price and its relationship with bubbles and their crashes, proving that the crashing of bubbles will increment the variation of the assets' price in the long-run.

Finally we gave an example that suggests that the stochastic economies admit bubble patterns that are considerably more diverse than the one observed in deterministic economies. The existence of an infinite number of periods in which crashes can occur is not only possible, it is completely compatible with the existence of bubbles in an infinite number of subtrees.

\section{Appendix A. Other proofs}

Proof of Proposition 1. The idea of the proof is to separate each set $A_{1}, A_{2}$ and $A_{3}$ to analyze them separately, and then, apply the dominated convergence theorem to have the result. For each set $A_{1}, A_{2, t_{1}, \ldots, t_{K}}^{K}, A_{3, t_{1}, t_{2} \ldots}$ and $\sigma$ belonging to any of the previous sets, we have that the analysis that can be done in the path $\sigma$ is analogous to the deterministic case with a, $\varepsilon$-contamination utility function (see Araujo et al. 4]). Therefore the results will be true for each path 
$\sigma$ that belongs to any of the sets described before.

Since the collection of all sets that have been described before is $2-2$ disjoint and non enumerable, there is an enumerable sub-collection with positive measure. Therefore we can rewrite the utility function in terms of these enumerable sub-collection only, and apply the deterministic case in each path that belongs to any of this subsets of the collection. Finally, if we apply the dominated convergence theorem (for a collection of generalized limits that are measurables in $\left.\left(\{1\} \times N^{\infty}, \mathcal{N}\right)\right)$, we conclude one part of the proof.

To prove the other part, notice that what we have done is to prove that the integral in $\sigma$ of elements of the super-differential of the utility function in each path $\sigma$, are in the super-differential of the utility function. And it can be easily observed that to prove second part is enough to prove that the super-differential is contained in the composition between the integral and the super-differential for each path.

Using some results of non differential analysis in Banach spaces, we have that, under the condition that we exposed before, the definition of supergradient and Clarke super-gradient are equivalents (see Clarke [10], page 36 Proposition 2.2.7). We also have that, under our hypothesis, the Clarke superdifferential of the utility function is contained in the integral in $\sigma$ of elements in the Clarke super-gradients of $\int_{\mathbb{N}} u \circ X_{\sigma_{t}} d \zeta_{i}(t)+\beta(\sigma) \inf _{t} u \circ X_{\sigma_{t}}$ for each $\sigma$ (see Clarke [10, page 76 Proposition 2.7.2). Which concludes the proof of the proposition.

Proof of Proposition 5 and Proposition 6 . Note that, for each node $s^{t}$, the optimal allocation is also efficient in the subtree generated by $s^{t}$. This subtree will be denoted by $s^{t}+$. Therefore there is $\left\{W^{i, s^{t}}\right\}_{i}$ new "endowment allocation" such that:

1. $\sum_{i} W_{s^{k}}^{i, s^{t}}=\sum_{i} W_{s^{k}}^{i} \forall s^{k}, \pi W^{i, s^{t}}=\pi W^{i} \forall i$ where $\pi$ is the AD price, 2. $W_{\sigma^{r}}^{i, s^{t}}=X_{\sigma^{r}}^{i}$ for all $\sigma$ and $r$ such that $r \leq t$ or $\sigma^{r}$ is not in the subtree 
generated by $s^{412}$ and

3. $W_{s^{t+k}}^{i, s^{t}}=W_{s^{t+k}}^{i} \forall k \geq 1$ for the rest of the endowment distributions in the subtree generated in $s^{t}$.

Note that for some nodes $s^{t}$ the new "endowments allocation"might be negative in some states of the economy for some agents. However, it does not imply that we cannot analyze the economy that it defines ${ }^{13}$ and its relationship with the original $\mathrm{AD}$ economy.

To establish this relationship, let us restrict the $\mathrm{AD}$ economy such that the agents maximize their consumption in the subtree generated by $s^{t}$ only, that is, the agent consumption set will be:

1. $\left\{X_{\sigma^{r}}^{i}\right\}$ for all path $\sigma$ and $r$ such that $r \leq t$ or $\sigma^{r}$ is not in the subtree generated by $s^{t}$, and

2. $\mathbb{R}_{+}$for all $s^{r}$ in the subtree generated by $s^{t}$.

Since the wealth of every agent and the efficient allocation are the same as their counterparts in the initial $\mathrm{AD}$ equilibrium, we have that the initial equilibrium price is in fact an equilibrium price for this restricted economy.

Let us analyze the stochastic sequential economy defined by the initial and the "new" endowment allocation for each $s^{t}$. To do so, let us define the new endowment distribution $\left\{\omega^{i, s^{t}}\right\}_{i}$ as $\omega^{i, s^{t}}=W^{i, s^{t}}-\sum_{j} R_{j} z_{j, s^{i}}^{i}$. Since the assets' prices are given by the Euler equations, and the FOC are the same in both cases, the prices are also the same in both economies. Using the same optimality conditions that were exposed in Araujo et al. (2019) (see [5]), we know that using the pure charge, $\nu_{i}^{s^{t}}$, the one that takes the highest value on the net trade $\left\{X^{i}-\omega^{i, s^{t}}\right\}$, we can implement the efficient allocation if we have

\footnotetext{
${ }^{12}$ This includes $\left\{s^{t,-(j)}\right\}_{j=1}^{t-1}$ all the predecessors of $s^{t}$.

${ }^{13}$ The economy defined by $\left\{W^{i, s^{t}}\right\}_{i}$ only differs with a standard economy on the fact that it may have a negative endowment in at most one date or event. In the case that you want to avoid the negative endowment in the node $s^{t}$, you can choose an endowment allocation $W_{s^{t}+k}^{i, s^{t}}$ such that $W_{s^{t}+k}^{i, s^{t}}>0$ for $k=0, \ldots, K^{s^{t}}$ and $W_{s^{t}+K+r}^{i, s^{t}}=W_{s^{t}+K+r}^{i}$ for $r \in \mathbb{N}$ satisfying the other conditions mentioned above.
} 


$$
\nu_{i}^{s^{t}}\left(X^{i}(z)-\omega^{i, s^{t}}\right) \leq \lim _{r}\left(\sum_{j} \sum_{s^{r,-(r-t)}=s^{t}} \mu_{s^{r}} q_{j, s^{r}} z_{j, s^{r}}\right)
$$

for every portfolio $z$, and with equality for $X^{i}$. Then, in order to implement sequentially this allocation, we have that

$\lim _{r} \sum_{s^{r,-(r-t)}=s^{t}} \sum_{j} \mu_{s^{r}} q_{j, s^{r}}=\sum_{j} z_{j, s^{t}}^{i} \nu\left(R_{j, s^{t}+}\right)+\nu_{i}^{s^{t}}\left(X^{i}-\omega^{i, s^{t}}\right)-\nu\left(X^{i}-\omega^{i, s^{t}}\right)$,

and since we have that

$$
\mu_{s^{t}} q_{j, s^{t}}=\sum_{r>t} \sum_{s^{r,-(r-t)}=s^{t}} \mu_{s^{r}} R_{s^{r}}^{j}+\lim _{r} \sum_{s^{r,-(r-t)}=s^{t}} \sum_{j} \mu_{s^{r}} q_{j, s^{r}}
$$

for each node $s^{t}$, the existence of a bubble in the economy at the state $s^{t}$ is given by the right part of Equation A.1. Finally, since the subtree generated by $s^{t}$ satisfies that $\left.x^{i}\right|_{\left[\sigma: \sigma_{t}=s^{t}\right]} \ggg 0$, Proposition 1 can be used restricted to this set proving that, under the conditions of Proposition 5, the pure charges in the super-gradient are null in this subtree, and that under the conditions of Proposition 6, the pure charges in the subtree are such that the right part of Equation A.1 is positive.

Remark 2. In Equation A.1 seems that the right part of the equality could be negative, something that does not make any sense since it would imply a negative bubble in the economy. However, this term is at least nonnegative due to the relationship between $\nu_{i}^{s^{t}}{ }^{14}$ and the rest of the pure charges that belong to the super-differential of $U^{i}$ when they are evaluated in the direction of the new "net trade", $X^{i}-\omega^{i, s^{t}}$. Moreover, if we sum Equation A.1 over the agents we have that

\footnotetext{
${ }^{14}$ The pure charge was chosen to be the pure charge with the highest value on the net trade $\left\{X^{i}-\omega^{i, s^{t}}\right\}$.
} 


$$
\begin{aligned}
I \lim _{r} \sum_{s^{r,-(r-t)}=s^{t}} \sum_{j} \mu_{s^{r}} q_{j, s^{r}} & =\sum_{i} \sum_{j} z_{j, s^{t}}^{i} \nu\left(R_{j, s^{t}+}\right)+\sum_{i} \nu_{i}^{s^{t}}\left(X^{i}-\omega^{i, s^{t}}\right) \\
& =\sum_{i} \sum_{j} z_{j, 0}^{i} \nu\left(R_{j, s^{t}+}\right)+\sum_{i} \nu_{i}^{s^{t}}\left(X^{i}-\omega^{i, s^{t}}\right) \\
& \geq 0
\end{aligned}
$$

since $\nu_{i}^{s^{t}}\left(X^{i}-\omega^{i, s^{t}}\right)-\nu\left(X^{i}-\omega^{i, s^{t}}\right) \geq 0$ for each $i$. Therefore, we have the following results.

Proof of Proposition 7 . Since:

- the bubble in the economy is characterized by the pure charges that exists in the super-gradient of the agent, more precisely given by A.1.

- the pure charges that exist in the super-gradient of the agents are integral in $\sigma$ of a collection of generalized limits, and

- the probability of each path, $\mathbb{P}(\{\sigma\})$, is zero;

we have that $\lim _{r} \sum_{s^{r,-(r-t)}=\sigma_{t}} \sum_{j} \mu_{s^{r}} q_{j, s^{r}} \rightarrow 0$ when $t \rightarrow \infty$.

Proof of Proposition 8. From the proof of Proposition 5 and 6, we know that the bubbles depends completely on the behavior of the pure charges in the superdifferential of the optimal allocation in the subtree generated by the analyzed node $\sigma_{t}$. Moreover, Proposition 1 ensures that Equation 5 is satisfied.

Under condition (1') or (2), we have that the right part of Equation A.1 is bounded from below by a positive constant multiplied by the capacity evaluated in the set $\left[\tilde{\sigma} \in A^{i}: \tilde{\sigma}_{r}=\sigma^{r}\right]$. Since $\mathbb{P}\left[A^{i}\right]>0$, for each $r \geq t$, there is $s^{r}$ such that $\mathbb{P}\left[\sigma \in A^{i}: \sigma_{r}=s_{r}\right] \geq \frac{\mathbb{P}\left[A^{i}\right]}{2|N| \mathbb{P}\left[\sigma: \sigma_{r}=s_{r}\right]}$ where $|N|$ is the number of elements in $N$, obtaining Equation 6 .

\section{References}

[1] O. J. Blanchard, Speculative bubbles, crashes and rational expectations, Economics letters 3 (4) (1979) 387-389. 
[2] O. J. Blanchard, M. W. Watson, Bubbles, rational expectations and financial markets (1982).

[3] M. S. Santos, M. Woodford, Rational asset pricing bubbles, Econometrica: Journal of the Econometric Society (1997) 19-57.

[4] A. Araujo, R. Novinski, M. R. Pascoa, General equilibrium, wariness and efficient bubbles, Journal of Economic Theory 146 (3) (2011) 785-811.

[5] A. Araujo, J. P. Gama, R. Novinski, M. R. Pascoa, Endogenous discounting, wariness, and efficient capital taxation, School of Economics Discussion Papers 06-19, University of Surrey. Conditionally accepted, Journal of Economic Theory.

[6] I. Gilboa, Expectation and variation in multi-period decisions, Econometrica: Journal of the Econometric Society (1989) 1153-1169.

[7] D. Schmeidler, Subjective probability and expected utility without additivity, Econometrica: Journal of the Econometric Society (1989) 571-587.

[8] T. F. Bewley, Existence of equilibria in economies with infinitely many commodities, Journal of Economic Theory 4 (3) (1972) 514-540.

[9] A. Mas-Colell, W. R. Zame, Equilibrium theory in infinite dimensional spaces, Handbook of mathematical economics 4 (1991) 1835-1898.

[10] F. H. Clarke, Optimization and nonsmooth analysis, Vol. 5, Siam, 1990. 